\section{The Transport of Rocks.}

May I ask Prof. Grenville A. J. Cole, through the medium of your columns, how far the authority for the statement that "the Portuguese stone ... was brought in carracks round the Cape to build the jutting fort on the coral shore of Moçambique" (NATURE, March 17, p. 353) is to be regarded as trustworthy?

I first saw this fort in $\mathrm{I} 9 \mathrm{II}$, and as recently as September last year I walked all round it. I have never been inside, but I am told by Portuguese residents on the island that the same kind of stone has been used throughout in the construction of the fort. This stone is a sandy coral-rock, with occasional small pebble bands. The country rock of Moçambique island is also a coral rock identical in composition and fossil contents - so far as one can judge by hand specimens and very numerous exposures-with that of which the fort is built. This material occurs in vast quantities on the eastern coast of Africa, and indeed on many tropical coast belts : it is well seen at Mombasa and Zanzibar, which island, like that of Moçambique, consists of little else. The coral-rock is not the best material for constructional purposes, as an examination of the external walls of the fort is sufficient to show. Can it be that this material was shipped all round the Cape ? It may be so, but I find it difficult to believe.

Fort Portal, Uganda, May 3. E. J. WAYLAND.

In reply to the interesting letter from $\mathrm{Mr}$. Wayland of the Geological Department of Uganda, I beg to say that my authority for the statement that the fort of Moçambique was built of stone brought from Portugal is the uninitialled article in the "Encyclopædia Britannica," I Ith ed., vol. I8, p. 949, where we read: "There are three forts, of which the principal, St. Sebastian, at the northern extremity of the island, was built in $x_{5} \mathrm{Jo}$ entirely of stone brought from Portugal."

I have examined the coral-rock here and at Mombasa, and, as Mr. Wayland states, it is not attractive for building purposes. I cannot speak as to the outer wall of the fort, and it may have been rebuilt or refaced since I $_{5}$ IO. It would be interesting now to pursue the matter in some detailed history of Moçambique.

Grenville A. J. Cole.

On Auroral Observations.

Ir has been found that the green auroral line is regularly visible in the clear night sky, and Lord Rayleigh has discovered the remarkable fact that it is more intense at Terling than in the north of England. A cognate investigation, which, so far as I know, has not yet been made, may be suggested to auroral observers, namely, to examine how the intensity changes at any one place throughout the night. The observation is doubtless a difficult one, but might be made by exposing a series of plates at different hours on a succession of clear nights. It would be of great interest to know whether or not the intensity remains nearly uniform throughout the night hours.

The University, Manchester,

S. Chapman, July 4 .

\section{Gradient of Potential near Electrodes.}

In NATURE of March 3I, p. 43I, Messrs. H. Nagaoka and $Y$. Sugiura describe a method of observing the Stark effect in the iron arc; namely, in the thin layer at the surface of the lower electrode. This fact points to the existence of a considerable gradient of potential in this layer.

In the course of an investigation of the radiation in the spark I have found, by means of direct electric measurements, the existence of a considerable gradient of potential in the thin layer that surrounds the electrodes when the sparking discharge takes place.

The discharge of 952 sparks per sec., yielding an effective current of 24 milliamperes, shows that the change of the difference of potential depends upon the length of sparks, as the accompanying diagram (Fig. I) shows. If the sparks are so short that the thin layers in the proximity of the electrodes, which yield a metallic spectrum, are not yet divided, then there exists a great gradient of potential (Fig. I, I). The size of gradient depends first upon the nature of the metal forming the electrodes. This is shown by the two curves on the diagram for electrodes. of platinum and aluminium.

At longer sparks, while among the above-mentioned layers only a spectrum of gas appears, the gradient of potential is much less (Fig. I, II); this does not depend upon the nature of the electrodes.

The intermediate space, marked by interrupted

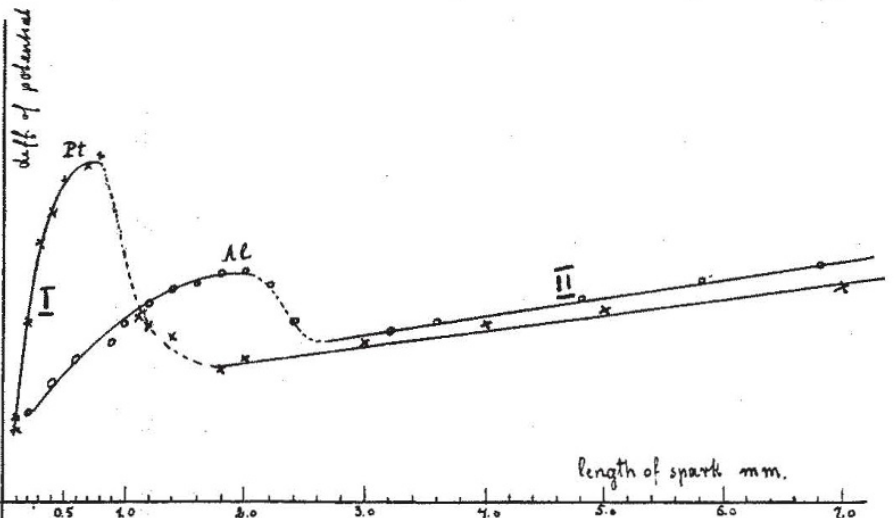

FIG. I.

lines, is difficult to examine. Sparks of both kinds generally come into view.

If $V_{1}$ signifies the difference of potential for short sparks, and $\mathrm{V}_{2}$ that for long sparks, we get for platinum and aluminium electrodes about :

$$
\left(\frac{\text { grad. } V_{1}}{\operatorname{grad} . V_{2}}\right)=\text { Io } \quad\left(\frac{\text { grad. } V_{1}}{\operatorname{grad} . V_{2}}\right)=5
$$

The thickness $x$ of the layer where there is a considerable gradient of potential is small :

$$
\begin{array}{ccc}
\text { Pt. } & \text { Cu. } & \text { Al. } \\
x=0.4 & 0.4-0.5 & 0.7-0.9 \mathrm{~mm} .
\end{array}
$$

This investigation is being continued.

Physical Institute,

University of Warsaw, Hoza 69.

S. Pieńkowski.

NO. 2803 , VOL. I I 27

\section{The Tides.}

The notice in NATURE of April I4, p. 508, of my pamphlet on the tides implies that I have completely misunderstood "the theory of the tide-generating force on the principle of gravitation." Regarding this I would like to present to your readers, very briefly, just one point in that theory: Newton, Herschel, and many other authorities compute the principal tide-raising force as the difference between the moon's attraction at the earth's centre and at the earth's surface. Now this is exactly the method 